\title{
Wegener's Granulomatosis with Laryngeal Location: A Case Report
}

\section{Mohamed Ali GLITI ${ }^{1,3 *}$, Khaoula Karim ${ }^{1,3}$, Bencheikh Razika ${ }^{2,3}$, Benbouzid Mohamed Anas²,3, Leila Essakalli} Houssyni $\mathrm{i}^{2,3}$

${ }^{1}$ Resident physician in otorhinolaryngology, Department of Otorhinolaryngology, Head and Neck Surgery, Ibn Sina University Hospital, Rabat, Morocco

${ }^{2}$ Professor of otorhinolaryngology, Department of Otorhinolaryngology, Head and Neck Surgery, Ibn Sina University Hospital, Rabat, Morocco

${ }^{3}$ Faculty of Medicine and Pharmacy of Rabat, Mohammed V University, Rabat, Morocco

ENT Department, Rabat Specialty Hospital, Ibn Sina University Hospital, Hafiane-Cherkaoui Avenue, 10100 Rabat, Morocco

DOI: $10.36347 /$ sjmcr.2020.v08i12.004

| Received: 19.01.2020 | Accepted: 26.01.2020 | Published: 05.12.2020

*Corresponding author: Mohamed Ali Gliti

Abstract

Objective: Describe the different therapeutic modalities of glottic stenosis due to Wegener's granulomatosis. Material and method: We report the case of a 49-year-old man who presented with a glottic localization of Wegener's granulomatosis. Clinical case: Mr. AB presented a laryngeal dyspnea on a laryngeal stenosis due to a Wegener's disease with renal, cutaneous, naso-sinus, and glottic localization. A life-saving tracheostomy was performed first. The medical treatment allowed a regression of all the lesions. . Two months after starting treatment, the patient is stable and the laryngeal stenosis no longer affects the patient's daily activities. Conclusion: The appearance of upper respiratory symptoms in may be indicative of Wegener's disease and should alert and seek for laryngo-tracheal stenosis. Intralesional corticosteroid injection is a recent and less invasive method than dilation. It seems to give satisfactory results. Sometimes a tracheostomy is necessary.

Key words: Wegner's granulomatosis, larynx tumor, dyspnea, tracheotomy.

Copyright $\odot 2020$ The Author(s): This is an open-access article distributed under the terms of the Creative Commons Attribution 4.0 International License (CC BY-NC 4.0) which permits unrestricted use, distribution, and reproduction in any medium for non-commercial use provided the original author and source are credited.

\section{INTRODUCTON}

Wegener's disease develops as necrotizing vasculitis affecting the small vessels. All the organs can be affected but the ENT, pulmonary and renal localizations are the most frequent $[1,2]$.

ENT manifestations are found in $92 \%$ of patients with wegner's disease with nasosinus involvement in $90 \%$ of cases, followed by otologic symptomatology in $40 \%$ of cases due to tubal dysfunction. Oropharyngeal localization is found in $10 \%$ of cases. The frequency of laryngeal localization is poorly understood. We report the case of a 49 -year-old patient with ENT localization wegner disease in the form of laryngeal involvement [1-4].

\section{Case Report}

It is about Mr A.B aged 49 and who presented to the ENT Emergency for an inspiratory dyspnea with notion of odynophagia and a dysphonia which goes back to two months of progressive aggravation, the whole evolving in a table asthenia, unencrypted fever, joint pain and intermittent bilateral nasal obstruction without any notion of rhinorhea or epistaxis. The patient has no personal or family history.

The nasofibroscopy objectified a bilateral atrophic rhinitis, withcrusts form inside the nose. At the laryngeal level: an inflamed aspect of the ventricular band and the arythenoide, as well as a mucous polyp at the level of the middle third of the left vocal cord, with mobility slightly diminished on the left side and preserved in the right side. The general examination finds a purpura in the lower right limb.

A cervico-thoracic CT scan with injection was carried out in the emergency room, and which objectified in addition to the polyp of the left vocal cord, a glottic stenosis at $1 \mathrm{~cm}$ from the vocal cords, taking approximately $80 \%$ of the circumference (stage III according to the classification of modified MeyersCotton), the pumonary radio is normal.

On the biological level, the patient presented an inflammatory syndrome with a CRP test at 83 , and a sedimentation rate at 40 associated with hypochromic microcytic anemia (hemoglobin at $10 \mathrm{~g} / \mathrm{dl}$ ). 
The patient received emergency corticotherapy (hydrocortisone hemisuccinate $120 \mathrm{mg}$ ) by vein, with an adrenaline nebulization without improvement, which motivated an emergency life-saving tracheostomy.

Direct suspension laryngoscopy was performed with biopsy of the polyp, the anatomopathological result of which is in favor of a non-specific inflammatory reaction. The dosage of angiotensin converting enzyme was normal, and the dosage of C-ANCA anti PR3 returned positive. The renal biopsy found a lesion of extracapillary glomerulonephritis.

All of the ENT, cutaneous and renal involvement associated with the inflammatory syndrome and the positivity of anti-PR3 C-ANCA allow us to retain Wegener's disease.

The treatment administered after the diagnosis was based on corticotherpy (oral prednisone $=1 \mathrm{mg} / \mathrm{kg}$ per day), combined with immunosuppressive treatment with intravenous cyclophosphamide (10 courses at a dose of $1100 \mathrm{mg}$ each). A prophylaxis antibiotic based on amoxicylline associated with clavulanic acid taken orally ( $1 \mathrm{~g}$ every 6 hours for 8 days) with abundant washing of the nasal cavities with physiological saline.

Two months after the intervention and the initiation of treatment, the patient reported a marked improvement in functional symptoms, with regression of dyspnea (at the temptation to withdraw from the tracheostomy) as well as odynophagia, dysphonia and nasal obstruction. The control nasofibroscopy finds a good permeation of the nasal cavities with a healthy mucous membranes and a regression of the glottic inflammation. The skin lesions and joint pain regressed; the CRP and the control blood count were normals.

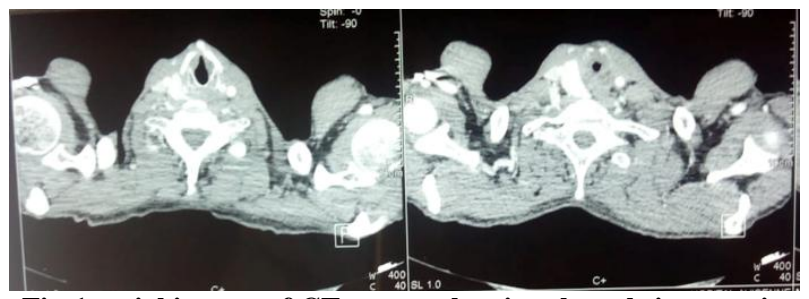

Fig-1: axial images of CT scan, a showing the golttic stenosis

\section{DISCUSSION}

Wegener's granulomatosis is a serious systemic disease of unknown origin characterized by its triple ENT, pulmonary and renal tropism. Its diagnosis is based on clinical, biological and histological arguments. The typical form of the disease involves systemic necrotizing angiitis, necrotic inflammation of the upper and lower airways, and extracapsular glomerulonephritis.
The etiopathogenesis of Wegner's granulomatosis remains debated. The fact of discovering the autoantibodies directed against the cytoplasm of the neutrophilic polymorphonuclear cells (ANCA) makes this disease considered as a true autoimmune disease. Infectious disease can also be blamed as a trigger for disease or relapse [3, 5]. Histologically, Wegener's granulomatosis combines three characteristic lesions: ischemic necrosis, giant cell granulomatosis and vasculitis that affect small and medium vessels. The average age at the time of diagnosis is between 40 and 50 years with extremes ranging from 9 to 78 years.

ENT involvement is present in $70 \%$ of patients with Wegener's granulomatosis and often revealing [4]. The symptoms presented are often non-specific, related to chronic rhinitis (nasal obstruction, crusts, epistaxis, etc.). Chronic sinusitis, the course of which is often interspersed with episodes of secondary infection, is also very common. Wegener's granulomatosis can also be accompanied by inflammatory damage to the outer ear (pavilion chondritis) and the middle ear with obstruction of the Eustachian tube [4]. The evolution can be towards a lysis of the middle ear leading to deafness and peripheral facial paralysis. Chronic ulcers of the oral mucosa, perforation of the palate, gingival enlargement or salivary and lacrimal glands are more rare manifestations of the disease.

Laryngeal involvement remains rare and appears to be more prevalent in women (4). It can initialize the table in 7 to $27 \%$ of cases (3).

The symptomatology of laryngeal localization, to which one could add the subglottic involvement associates dyspnea, dysphonia, stridor and cough $[8,13$, 14]. Indirect laryngoscopy or nasofibroscopy visualizes stenosis and appreciates its location, extent, fibrous or inflammatory appearance and the importance of reducing the tracheal lumen.

It is important, even compulsory, to assess the impact of laryngo tracheal stenosis on daily life and to judge its severity. This impact is mainly assessed by interrogation and clinical examination [8]. The damage can be fatal as in the case reported by Matt [3]. Laryngo tracheal stenosis in Wegener's granulomatosis can persist despite the control of other localizations by medical treatment and evolve independently of the others [8].

The emergency cervicothoracic scanner provides information on the state of the larynx: especially cricoid cartilage which can be lysed. It appreciates the extent of the lesions, and guides the exact location of the tracheostomy. CT is important to assess the initial lesions in order to be able to judge the possibilities of performing a tracheal anastomosis resection. Functional respiratory tests (flow-volume 
curve) make it possible to confirm the presence of a tracheal obstacle and to follow the evolution after treatment.

The purpose of laryngo-tracheal endoscopy under general anesthesia is to accurately assess the characteristics of the stenosis: its location, its extent, its fibrous or inflammatory appearance and the reduction of the tracheal lumen and make a good mapping of the lesions as well as guide the biopsies.

$\begin{array}{rrr}\text { Laryngo tracheal } & \text { stenosis is usually treated } \\ \text { with } & \text { glucocorticoids } & \text { associated }\end{array}$ immunosupressants and allows its regression, but the success rate is low varying from 20 to $26 \%$ according to the authors $[1,6]$.

If the medical treatment is not effective or if the laryngotracheal stenosis is isolated, local treatment is therefore necessary [8].

There are many surgical options ranging from endoscopic treatment to laryngotracheoplasty $[1,8]$. A tracheostomy may be necessary or even urgent for some patients. Lebovic et al. reported a $52 \%$ incidence of transient tracheostomy [1]. However, the development of endoscopic techniques seems to decrease the incidence of this gesture $[8,14]$.

Endoscopic procedures include resection of the laser stenosis, tracheal dilations, and local application of mitomycin and intralesional corticosteroid injection [8, 14]. The vaporization of the laryngo-tracheal stenosis with the laser can be complicated at an early stage (within ten to 21 days following the gesture) by a recurrence, sometimes more extensive $[1,8]$. In our reported cases, the patient has had no recurrence within 20 days of the initial treatment.

The intra-lesional injections of methylprednisolone associated with tracheal dilation reported by Hoffman et al. have reported several successes, making this therapy a choice of first intention for laryngo-tracheal stenosis. reducing the tracheal lumen by more than $50 \%$ [1,11,12].Langford et al. perform this technique in the event of respiratory repercussions with a tracheal diameter less than 5 or 4 $\mathrm{mm}$ depending on the sex [8]. These injections are performed in the submucosa at the level of the four quadrants of the stenosis. The total dose injected ranges from 40 to $120 \mathrm{mg}$ of methylprednisolone [1, 14]. However, it is noted by users, a $50 \%$ leak of corticosteroid during the procedure. These authors have shown good efficacy of this technique with the absence of tracheostomy performed during the management of 12 laryngo-tracheal stenosis for patients with Wegner's granulomatosis. Pneumothorax is the main complication of this therapy $[8,14]$. Thus, it is necessary to favor the least traumatic techniques possible for stenosis [8, 14]. Even if the current trend is for intra-lesional injection of corticosteroids in the area of laryngo-tracheal stenosis, the indications for the various local treatments are not codified. Thus, the choice of treatment depends on the experience of each center.

The surveillance of Wegener's granulomatosis is attentive, close and prolonged. It has three objectives: evolution of the symptomatology, recurrence of the disease and monitoring of the undesirable effects of the treatments. For laryngotracheal stenosis, the impact on the daily and sports activities of the patients is evaluated. Biological examinations (determination of cANCA, CRP, proteinuria) will allow the relapse of the disease to be detected. Finally, these surveillance consultations will verify the absence of undesirable effects from long-term drugs (corticosteroid therapy and immunosuppressants) $[9,10]$.

\section{CONCLUSiON}

Glottic subglottic involvement of patients with Wegener's granulomatosis is uncommon and rarely reported. The appearance of dysphonia, cough or dyspnea should motivate an ENT consultation, and managed quickly. The diagnosis is made by indirect laryngoscopy or nasofibroscopy. Failure of immunosuppressive medical treatment should prompt surgical management. Intra-lesional corticosteroid injection is a recent method, the results of which appear satisfactory.

\section{REFERENCES}

1. Lebovics RS, Hoffman GS, Leavitt RY, Kerr GS, Hallahan C, Rottem M, Fauci AS, Travis WD, Kammerer W. The management of subglottic stenosis in patients with Wegener's granulomatosis. The Laryngoscope. 1992 Dec;102(12):1341-5.

2. Kornblut AD, Wolff SM, DeFries HO, Fauci AS. Wegener's granulomatosis. Laryngoscope. 1980; 90:1453-65.

3. Matt B. Wegener's granulomatosis, acute laryngotracheal airway obstruction and death in a 17-year-old female: case report and review of the litterature. Int J Pediatr Otolaryngol. 1997; 37:16372.

4. Gluth M, Shinners P, Kasperbauer J. Subglottic stenosis associated with Wegener's granulomatosis. Laryngoscope. 2003; 113:1304-7.

5. Djalilian M, McDonald TJ, Devine KD, Weiland LH. Nontraumatic, nonneoplastic subglottic stenosis. Ann Otol Rhinol Laryngol. 1975; 84:75763.

6. Lee CC, Singer AJ. Respiratory failure due to subglottic stenosis from relapsing polychondritis. Is J Emerg Med. 2006; 24:750-2.

7. Alaani A, Hogg P, Drake Lee AB. Wegener's granulomatosis and subglottic stenosis: 
management of the airway. J Laryngol Otol. 2004; 118:786-90.

8. Langford C, Sneller M, Hallahan C. Clinical features and therapeuric management of subglottic stenosis in patient with Wegener's granulomatosis. Arthritis Rheum. 1996; 10:1754-60.

9. Puechal X. Antineutrophil cytoplasmic antibodyassociated vasculitides. Joint Bone Spine. 2007; 74(5):427-35.

10. Belmont HM. Treatment of ANCA-associated systemic vasculitis. Bull NYHU Hosp Jt Dis 2006; 64:60-6.

11. Daum TE, Specks U, Colby TV, Edell ES, Brutinel MW, Prakash UB. Tracheobronchial involvement in Wegener's granulomatosis. Is J Respir Crit Care
Med. 1995; 151:522-6.

12. Costentin B, Dehesdin D, Marie JP, Scarcellalecler V, Andrieu-Guitancourt J. Granulomatose de Wegener et localisations ORL. Analyse retrospective de 16 patients. Ann Otolaryngol Chir Cervicofac. 2001; 118:306-14.

13. Stappaerts I, Van Laer C, Deschepper K, Van de Heyning P, Vermeire. Endoscopic management of severe subglottic stenosis in Wegener's granulomatosis. Clin Rheumatol. 2001; 19:315-7.

14. Hoffman G, Thomas-Golbanov C, Chan J, Akst L, Eliachar I. Treatment of subglottic stenosis, due to Wegener's granulomatosis, with intralesional corticosteroid and dilatation. J Reumatology. 2003;30:1017-2. 